\title{
Learning Commonalities in SPARQL
}

\author{
Sara El Hassad, François Goasdoué, and Hélène Jaudoin \\ IRISA, Univ. Rennes 1, Lannion, France
}

\begin{abstract}
Finding the commonalities between descriptions of data or knowledge is a foundational reasoning problem of Machine Learning. It was formalized in the early 70's as computing a least general generalization (lgg) of such descriptions. We revisit this well-established problem in the SPARQL query language for RDF graphs. In particular, and by contrast to the literature, we address it for the entire class of conjunctive SPARQL queries, a.k.a. Basic Graph Pattern Queries (BGPQs), and crucially, when background knowledge is available as RDF Schema ontological constraints, we take advantage of it to devise much more precise lggs, as our experiments on the popular DBpedia dataset show.
\end{abstract}

Keywords: BGP queries $\cdot$ RDF $\cdot$ RDFS $\cdot$ least general generalization

\section{Introduction}

Finding the commonalities between descriptions of data or knowledge is a fundamental Machine Learning problem, which was formalized in the early 70's as computing a least general generalization (lgg) of such descriptions [21]. Since then, it has also received consideration in the Knowledge Representation field, where least general generalizations were rebaptized least common subsumers [5], in Description Logics $[1,5,14,27]$ and in Conceptual Graphs [3]. More recently, this problem started being investigated in RDF $[7,9]$ and its associated SPARQL query language $[2,10,16]$, the two prominent Semantic Web standards by W3C. Motivations. We study this old reasoning problem in the SPARQL setting (contributions to be outlined shortly), i.e., when input descriptions are SPARQL queries. Solutions to this problem can be applied to a variety of useful important applications, ranging from optimization to exploration and recommendation in RDF data management systems or in SPARQL endpoints. For instance, an lgg of incoming queries characterizes the largest set of their commonalities whose processing may be shared in multi-query optimization [15]. Similarly, Iggs of subsets of a query workload correspond to candidate views that may be recommended for materialization in view selection [11], a typical optimization for data warehouses [6], and among which can be selected those that allow rewriting (partially or totally) the workload while minimizing a combination of rewriting processing, view storage and view maintenance costs. Also, clustering user queries found in system logs, based on their lggs, may help classifying the queries and identifying the kind of data each category accesses [4]. Finally, finding the relevant user query cluster for an incoming query may help recommending similar and complementary searches [13]. 
Contributions. We bring the following contributions to the problem of finding an lgg of SPARQL queries:

1. We carefully study a novel notion of lgg for the popular conjunctive fragment of SPARQL (Section 3), a.k.a. Basic Graph Pattern Queries (BGPQs). Our definition, which we briefly outlined in [10], significantly departs from the literature by $(i)$ considering general BGPQs, instead of unary tree-shaped ones [2, 16], and crucially by (ii) taking advantage of background knowledge formalized as RDF Schema (RDFS) ontological constraints. Furthermore, to establish this definition of an lgg, we revise the standard generalization/specialization relation (a.k.a. entailment) between BGPQs in order to devise a well-founded entailment relation that allows comparing BGPQs w.r.t. extra RDFS constraints, i.e., the counterpart to subsumption between concepts w.r.t. a terminology in Description Logics and to containment between queries w.r.t. constraints in Databases.

2. We provide a solution to the above problem (Section 4 ), which technically differs from the state of the art $[2,16]$ in that it cannot exploit the (imposed) tree-shape of the input BGPQs to compute their lgg through a simultaneous root-to-leaves traversal. Instead, our solution traverses blindly the general (hence arbitrary-shaped) input BGPQs and builds their lgg using the notion of least general anti-unification of atoms [21,23], which is dual to the well-known notion of most general unification of atoms [22,23]. Also, to take into account background knowledge, we define a well-founded notion of saturation of BGPQs w.r.t. extra RDFS constraints, which we devise inspired by that of RDF graphs.

3. We report on experiments made to assess the added-value of considering background knowledge when computing lggs of BGPQs (Section 5). Notably, we use real data from DBpedia to show how much more precise lggs are when background knowledge is considered, by measuring the gain in precision it yields. Organization. Following the presentation of [8-10], we first recall the basics of RDF and SPARQL in Section 2. Then, we detail the above contributions. Finally, we discuss related work and conclude in Section 6.

Supplementary material (proofs of our technical results, implemented algorithms, additional experiments, etc) is available in our online research report [8].

\section{Preliminaries}

\subsection{The Resource Description Framework (RDF)}

RDF graphs. The RDF data model allows specifying RDF graphs. An RDF graph is a set of triples of the form $(\mathrm{s}, \mathrm{p}, \mathrm{o})$. A triple states that its subject $\mathbf{s}$ has the property $\mathrm{p}$, the value of which is the object $\mathrm{o}$. Triples are built using three pairwise disjoint sets: a set $\mathcal{U}$ of uniform resources identifiers (URIs), a set $\mathcal{L}$ of literals (constants), and a set $\mathcal{B}$ of blank nodes allowing to support incomplete information. Blank nodes are identifiers for missing values (unknown URIs or literals). Well-formed triples, as per the RDF specification [24], belong to $(\mathcal{U} \cup \mathcal{B}) \times \mathcal{U} \times(\mathcal{U} \cup \mathcal{L} \cup \mathcal{B})$; we only consider such triples hereafter.

Notations. We use $\mathbf{s}, \mathrm{p}$, o in triples as placeholders. We note $\operatorname{Val}(\mathcal{G})$ the set of values occurring in an RDF graph $\mathcal{G}$, i.e., the URIs, literals and blank nodes; 


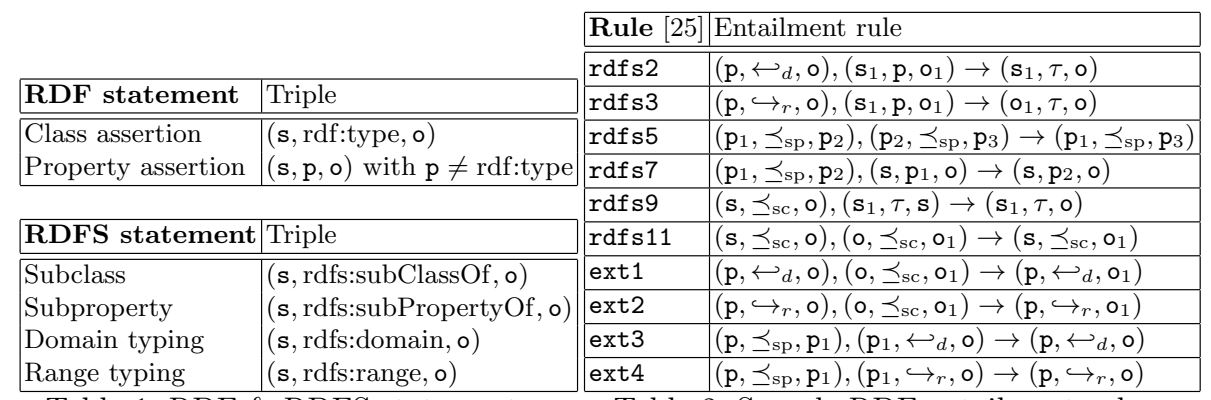

Table 1: RDF \& RDFS statements. Table 2: Sample RDF entailment rules.
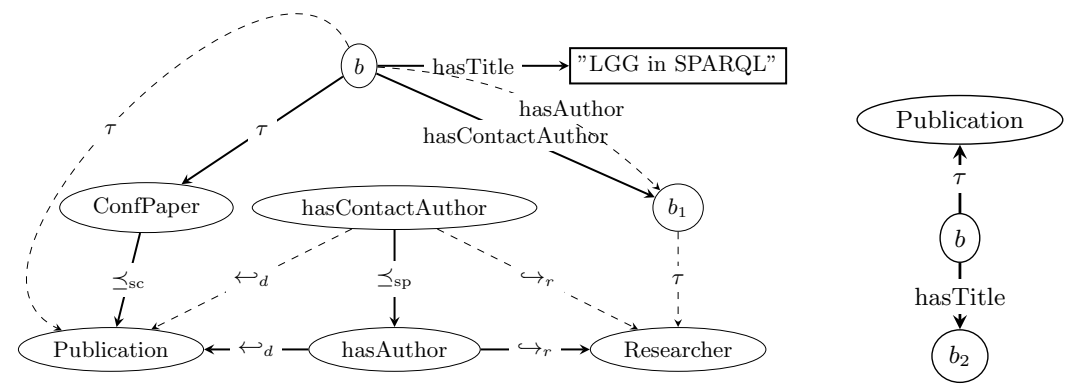

Fig. 1: Sample RDF graph $\mathcal{G}$

Fig. 2: Sample RDF graph $\mathcal{G}^{\prime}$.

we note $\mathrm{Bl}(\mathcal{G})$ the set of blank nodes occurring in $\mathcal{G}$. A blank node is written $b$ possibly with a subscript, and a literal is a string between quotes. For instance, the triples ( $b$, hasTitle, "LGG in SPARQL") and ( $b$, hasContactAuthor, $\left.b_{1}\right)$ mean: something (b) entitled " $L G G$ in SPARQL" has somebody $\left(b_{1}\right)$ as contact author.

A triple models an assertion, either for a class (unary relation) or for a property (binary relation). Table 1 (top) shows the use of triples to state such assertions. The RDF standard [24] provides built-in classes and properties, as URIs within the rdf and rdfs pre-defined namespaces, e.g., rdf:type which can be used to state that the above $b$ is a conference paper with the triple (b, rdf:type, ConfPaper).

Adding ontological knowledge to RDF graphs. An essential feature of $\mathrm{RDF}$ is the possibility to enhance the descriptions in RDF graphs by declaring ontological constraints between the classes and properties they use. This is achieved with RDF Schema (RDFS) statements, which are triples using particular built-in properties. Table 1 (bottom) lists the allowed constraints and the triples to state them; domain and range denote respectively the first and second attribute of every property. For example, the triple (ConfPaper, rdfs:subClassOf, Publication) states that conference papers are publications, the triple (hasContactAuthor, rdfs:subPropertyOf, hasAuthor) states that having a contact author is having an author, the triple (hasAuthor, rdfs:domain, Publication) states that only publications may have authors, and the triple (hasAuthor, rdfs:range, Researcher) states that only researchers may be authors of something.

Notations. For conciseness, we use the following shorthands for RDFS builtin properties: $\tau$ for rdf:type, $\preceq_{\text {sc }}$ for rdfs:subClassOf, $\preceq_{\text {sp }}$ for rdfs:subPropertyOf, $\hookleftarrow_{d}$ for rdfs:domain, and $\hookrightarrow_{r}$ for rdfs:range. 
Figure 1 displays the usual representation of the RDF graph $\mathcal{G}$ made of the seven above-mentioned triples, which are called the explicit triples of $\mathcal{G}$. A triple $(\mathrm{s}, \mathrm{p}, \mathrm{o})$ corresponds to a $\mathrm{p}$-labeled directed edge from the $\mathrm{s}$ node to the o node: $\mathrm{s} \stackrel{\mathrm{p}}{\rightarrow} \mathrm{o}$. Explicit triples are shown as solid edges, while the implicit ones, which are derived using ontological constraints (see below), are shown as dashed edges.

Importantly, it is worth noticing the deductive nature of ontological constraints, which begets implicit triples within an RDF graph. For instance, in Figure 1, the constraint (hasContactAuthor, $\preceq_{\mathrm{sp}}$, hasAuthor) together with the triple ( $b$, hasContactAuthor, $b_{1}$ ) imply the implicit triple ( $b$, hasAuthor, $\left.b_{1}\right)$, which, further, with the constraint (hasAuthor, $\hookrightarrow_{r}$, Researcher) yields another implicit triple $\left(b_{1}, \tau\right.$, Researcher).

Deriving the implicit triples of an RDF graph. The RDF standard defines a set of entailment rules in order to derive automatically all the triples that are implicit to an RDF graph. Table 2 shows the strict subset of these rules that we will use to illustrate important notions as well as our contributions in the next sections; importantly, our contributions hold for the entire set of entailment rules of the RDF standard, and any subset of thereof. The rules in Table 2 concern the derivation of implicit triples using ontological constraints (i.e., RDFS statements). They encode the propagation of assertions through constraints ( $\operatorname{rdf} \mathrm{s} 2$, $\operatorname{rdfs} 3, \operatorname{rdfs} 7, \operatorname{rdfs} 9)$, the transitivity of the $\preceq_{\mathrm{sp}}$ and $\preceq_{\mathrm{sc}}$ constraints (rdfs5, rdfs11), the complementation of domains or ranges through $\preceq_{\mathrm{sc}}$ (ext1, ext2), and the inheritance of domains and of ranges through $\preceq_{\mathrm{sp}}$ (ext3, ext4).

The saturation (or closure) of an RDF graph $\mathcal{G}$ w.r.t. a set $\mathcal{R}$ of RDF entailment rules (a.k.a. entailment regime) is the RDF graph $\mathcal{G}^{\infty}$ obtained by adding to $\mathcal{G}$ all the implicit triples that follow from $\mathcal{G}$ and $\mathcal{R}$. Roughly speaking, the saturation $\mathcal{G}^{\infty}$ materializes the semantics of $\mathcal{G}$. It corresponds to the fixpoint reached by repeatedly applying the rules in $\mathcal{R}$ to $\mathcal{G}$ in a forward-chaining fashion, while adding to $\mathcal{G}$ the triples they derive. In RDF, the saturation is finite, unique (up to blank node renaming), and can be computed in polynomial time [25].

The saturation of the RDF graph $\mathcal{G}$ shown in Figure 1 corresponds to the $\mathrm{RDF}$ graph $\mathcal{G}^{\infty}$ in which all the $\mathcal{G}$ implicit triples (dashed edges) are made explicit (solid edges). It is worth noting how, starting from $\mathcal{G}$, applying RDF entailment rules mechanizes the construction of $\mathcal{G}^{\infty}$. For instance, recall the reasoning sketched above for deriving the triple $\left(b_{1}, \tau\right.$, Researcher). This is automated by the following sequence of applications of RDF entailment rules: (hasContactAuthor, $\preceq_{\mathrm{sp}}$, hasAuthor) and ( $b$, hasContactAuthor, $b_{1}$ ) trigger rdf $\mathrm{s} 7$ that adds $\left(b\right.$, hasAuthor, $\left.b_{1}\right)$ to the RDF graph. In turn, this new triple together with (hasAuthor, $\hookrightarrow_{r}$, Researcher) triggers rdfs3 that adds $\left(b_{1}, \tau\right.$, Researcher).

Comparing RDF graphs. The RDF standard defines a generalization/specialization relationship between two RDF graphs, called entailment between graphs. Roughly speaking, an RDF graph $\mathcal{G}$ is more specific than another RDF graph $\mathcal{G}^{\prime}$, or equivalently $\mathcal{G}^{\prime}$ is more general than $\mathcal{G}$, whenever there is an embedding of $\mathcal{G}^{\prime}$ into the saturation of $\mathcal{G}$, i.e., the complete set of triples that $\mathcal{G}$ models.

More formally, given any subset $\mathcal{R}$ of RDF entailment rules, an RDF graph $\mathcal{G}$ entails an RDF graph $\mathcal{G}^{\prime}$, denoted $\mathcal{G} \models_{\mathcal{R}} \mathcal{G}^{\prime}$, iff there exists an homomorphism 
$\phi$ from $\operatorname{Bl}\left(\mathcal{G}^{\prime}\right)$ to $\operatorname{Val}\left(\mathcal{G}^{\infty}\right)$ such that $\left[\mathcal{G}^{\prime}\right]_{\phi} \subseteq \mathcal{G}^{\infty}$, where $\left[\mathcal{G}^{\prime}\right]_{\phi}$ is the $\mathrm{RDF}$ graph obtained from $\mathcal{G}^{\prime}$ by replacing every blank node $b$ by its image $\phi(b)$.

Figure 2 shows an RDF graph $\mathcal{G}^{\prime}$ entailed by the RDF graph $\mathcal{G}$ in Figure 1 w.r.t. the entailment rules displayed in Table 2. In particular, $\mathcal{G} \models_{\mathcal{R}} \mathcal{G}^{\prime}$ holds for the homomorphism $\phi$ such that: $\phi(b)=b$ and $\phi\left(b_{2}\right)=$ "LGG in SPARQL". By contrast, when $\mathcal{R}$ is empty, this is not the case (i.e., $\mathcal{G} \nvdash_{\mathcal{R}} \mathcal{G}^{\prime}$ ), as the dashed edges in $\mathcal{G}$ are not materialized by saturation, hence the $\mathcal{G}^{\prime}$ triple ( $b, \tau$, Publication) cannot have an image in $\mathcal{G}$ through some homomorphism.

Notations. When relevant to the discussion, we designate by $\mathcal{G} \models_{\mathcal{R}}^{\phi} \mathcal{G}^{\prime}$ the fact that the entailment $\mathcal{G}=_{\mathcal{R}} \mathcal{G}^{\prime}$ holds due to the graph homomorphism $\phi$. Also, when $\mathrm{RDF}$ entailment rules are disregarded, i.e., $\mathcal{R}=\emptyset$, we note the entailment relation $=$ (without indicating the rule set at hand).

Importantly, from the definition of entailment between two RDF graphs [24, 25], the following holds:

Property 1. Given two RDF graphs $\mathcal{G}, \mathcal{G}^{\prime}$ and a set $\mathcal{R}$ of RDF entailment rules, (i) $\mathcal{G}$ and $\mathcal{G}^{\infty}$ are equivalent $\left(\mathcal{G} \models_{\mathcal{R}} \mathcal{G}^{\infty}\right.$ and $\mathcal{G}^{\infty} \models_{\mathcal{R}} \mathcal{G}$ hold), noted $\mathcal{G} \equiv_{\mathcal{R}} \mathcal{G}^{\infty}$, and (ii) $\mathcal{G} \models_{\mathcal{R}} \mathcal{G}^{\prime}$ holds iff $\mathcal{G}^{\infty} \models \mathcal{G}^{\prime}$ holds.

From a practical viewpoint, Property 1 points out that checking $\mathcal{G} \models_{\mathcal{R}} \mathcal{G}^{\prime}$ can be done in two steps: a reasoning step that computes the saturation $\mathcal{G}^{\infty}$ of $\mathcal{G}$, followed by a standard graph homomorphism step that checks if $\mathcal{G}^{\infty} \models \mathcal{G}^{\prime}$ holds.

\subsection{SPARQL Conjunctive Queries}

Basic graph pattern queries. The well-established conjunctive fragment of SPARQL queries, a.k.a. Basic Graph Pattern queries (BGPQs), is the counterpart of the select-project-join queries for databases; it is the most widely used subset of SPARQL queries in real-world applications [19].

A Basic Graph Pattern (BGP) is a set of triple patterns, or simply triples by a slight abuse of language. They generalize RDF triples by allowing the use of variables. Given a set $\mathcal{V}$ of variables, pairwise disjoint with $\mathcal{U}, \mathcal{L}$ and $\mathcal{B}$, triple patterns belong to: $(\mathcal{V} \cup \mathcal{U} \cup \mathcal{B}) \times(\mathcal{V} \cup \mathcal{U}) \times(\mathcal{V} \cup \mathcal{U} \cup \mathcal{L} \cup \mathcal{B})$.

Notations. We adopt the usual conjunctive query notation $q(\bar{x}) \leftarrow t_{1}, \ldots, t_{\alpha}$, where $\left\{t_{1}, \ldots, t_{\alpha}\right\}$ is a BGP. The head of $q$, noted head $(q)$, is $q(\bar{x})$, and the body of $q$, noted $\operatorname{body}(q)$, is the BGP $\left\{t_{1}, \ldots, t_{\alpha}\right\}$ the cardinality of which is the size of $q$. The query head variables $\bar{x}$ are called answer variables, and form a subset of the variables occurring in $t_{1}, \ldots, t_{\alpha}$; for Boolean queries, $\bar{x}$ is empty. The cardinality of $\bar{x}$ is the arity of $q$. We use $x$ and $y$ in queries, possibly with subscripts, for answer and non-answer variables respectively. Finally, we note $\operatorname{VarBl}(q)$ the set of variables and blank nodes occurring in the query $q$, and $\operatorname{Val}(q)$ the set of all its values, i.e., URIs, blank nodes, literals and variables.

Entailing and answering queries. Two related important notions characterize how an RDF graph contributes to a query.

The weaker notion, called query entailment, indicates whether or not an RDF graph holds some answer(s) to a query. It generalizes entailment between RDF 
graphs, to account for the presence of variables in the query body, for establishing whether an RDF graph entails a query, i.e., whether the query embeds in that graph. Formally, given a BGPQ $q$, an RDF graph $\mathcal{G}$ and a set $\mathcal{R}$ of RDF entailment rules, $\mathcal{G}$ entails $q$, noted $\mathcal{G} \models_{\mathcal{R}} q$, iff $\mathcal{G} \models_{\mathcal{R}} \operatorname{bod}(q)$ holds, i.e., there exists a homomorphism $\phi$ from $\operatorname{VarBl}(q)$ to $\operatorname{Val}\left(\mathcal{G}^{\infty}\right)$ such that $[\operatorname{body}(q)]_{\phi} \subseteq \mathcal{G}^{\infty}$.

The RDF graph $\mathcal{G}$ in Figure 1 entails the query $q\left(x_{1}, x_{2}\right) \leftarrow\left(x_{1}, \tau, x_{2}\right)$ asking for all the resources and their classes for instance, because of the homomorphism $\phi$ such that $\phi\left(x_{1}\right)=b$ and $\phi\left(x_{2}\right)=$ ConfPaper. Observe that this entailment holds for any subset of RDF entailment rules, since the above homomorphism $\phi$ already holds for $\mathcal{R}=\emptyset$, i.e., considering only the explicit triples in Figure 1 .

Notations. Similarly to entailment between RDF graphs, we denote by $\mathcal{G} \models_{\mathcal{R}}^{\phi} q$ that the entailment $\mathcal{G} \models_{\mathcal{R}} q$ holds due to the homomorphism $\phi$.

The stronger notion characterizing how an RDF graph contributes to a query, called query answering, identifies all the query answers that this graph holds. Formally, given a BGPQ $q$ with set $\bar{x}$ of answer variables, the answer set of $q$ against $\mathcal{G}$ is $q(\mathcal{G})=\left\{(\bar{x})_{\phi} \mid \mathcal{G} \models_{\mathcal{R}}^{\phi}\right.$ body $\left.(q)\right\}$, where $(\bar{x})_{\phi}$ is the tuple of $\mathcal{G}^{\infty}$ values obtained by replacing every answer variable $x_{i} \in \bar{x}$ by its image $\phi\left(x_{i}\right)$. In case of a Boolean query, $q$ is false iff $q(\mathcal{G})=\emptyset$; otherwise $q$ is true and $q(\mathcal{G})=\{\langle\rangle\}$ where \langle\rangle denotes the empty tuple.

The answer set to the above query $q\left(x_{1}, x_{2}\right) \leftarrow\left(x_{1}, \tau, x_{2}\right)$ against the RDF graph $\mathcal{G}$ in Figure 1 is:

- $\left\{\langle b\right.$, ConfPaper $\rangle,\langle b$, Publication $\rangle,\left\langle b_{1}\right.$, Researcher $\left.\rangle\right\}$ for $\mathcal{R}$ the set of entailment rules in Table 2, i.e., considering the explicit and implicit triples in Figure 1;

- $\{\langle b$, ConfPaper $\rangle\}$ for $\mathcal{R}=\emptyset$, i.e., considering only the explicit triples in Figure 1.

Importantly, from the definition of answer set of a SPARQL query against an RDF graph [26], the following holds:

Property 2. Given an RDF graph $\mathcal{G}$, a set $\mathcal{R}$ of entailment rules and a BGPQ $q$, (i) $\mathcal{G} \models_{\mathcal{R}} q$ holds iff $\mathcal{G}^{\infty} \mid=q$ holds, and (ii) $q(\mathcal{G})=q\left(\mathcal{G}^{\infty}\right)$ holds.

From a practical viewpoint, Property 2 points out that query entailment $\mathcal{G} \models_{\mathcal{R}} q$, respectively query answering $q(\mathcal{G})$, can be done in two steps: a reasoning step that computes the saturation $\mathcal{G}^{\infty}$ of $\mathcal{G}$, followed by a standard graph homomorphism step that checks if $\mathcal{G}^{\infty} \models^{\phi} q$ holds for some homomorphism $\phi$, respectively enumerates all the homomorphisms $\phi$ for which $\mathcal{G}^{\infty} \models^{\phi} q$ holds.

Comparing queries. Similarly to RDF graphs, queries can be compared through the generalization/specialization relationship of entailment between queries.

Let $q, q^{\prime}$ be BGPQs with the same arity, whose heads are $q(\bar{x})$ and $q^{\prime}\left(\bar{x}^{\prime}\right)$, and $\mathcal{R}$ the set of RDF entailment rules under consideration. $q$ entails $q^{\prime}$, denoted $q \models_{\mathcal{R}} q^{\prime}$, iff $\operatorname{bod} y(q) \models_{\mathcal{R}}^{\phi} \operatorname{bod} y\left(q^{\prime}\right)$ with $\left(\bar{x}^{\prime}\right)_{\phi}=\bar{x}$ holds. Here, $\operatorname{bod} y(q) \models_{\mathcal{R}}^{\phi} \operatorname{body}\left(q^{\prime}\right)$ is the adaptation of the above-mentioned entailment relationships between RDF graphs to the fact that the query bodies may feature variables, i.e., $\phi$ is a homomorphism from $\operatorname{Var} B l\left(\operatorname{bod} y\left(q^{\prime}\right)\right)$ to $\operatorname{Val}\left(\operatorname{bod} y(q)^{\infty}\right)$ such that $\left[\operatorname{body}\left(q^{\prime}\right)\right]_{\phi} \subseteq$ $\operatorname{bod} y(q)^{\infty}$; the saturation of a BGP body, here $\operatorname{bod} y(q)^{\infty}$, is the obvious generalization of RDF graph saturation that treats variables as blank nodes, since they both equivalently model unknown information within BGPs [26]. 
For instance, the query $q_{1}(x) \leftarrow(x, \tau$, ConfPaper $)$, ( $x$, hasContactAuthor, $\left.y\right)$ entails the query $q_{2}(x) \leftarrow(x, \tau, y)$ with $\phi(x)=x, \phi(y)=$ ConfPaper and any set of entailment rules.

We remark that entailment between queries, query entailment and query answering (obviously) relate as follows:

Property 3. Given an RDF graph $\mathcal{G}$, a set $\mathcal{R}$ of entailment rules and two BGPQs $q, q^{\prime}$ such that $q \models_{\mathcal{R}} q^{\prime},(i)$ if $\mathcal{G} \models_{\mathcal{R}} q$ holds then $\mathcal{G} \models_{\mathcal{R}} q^{\prime}$ holds, and (ii) $q(\mathcal{G}) \subseteq q^{\prime}(\mathcal{G})$ holds.

Finally, query entailment, query answering and entailment between queries treat blank nodes in queries exactly as non-answer variables [26]. Hence, hereafter, we assume without loss of generality that queries do not use blank nodes.

\section{Problem Statement}

A least general generalization (lgg) of $n$ descriptions $d_{1}, \ldots, d_{n}$ is a most specific description $d$ generalizing $d_{1}, \ldots, d_{n}$ for some generalization/specialization relation [21]. In our SPARQL setting, we may use off-the-shelf BGPQs as descriptions and entailment between BGPQs as generalization/specialization relation:

Definition 1 (lgg of BGPQs). Let $q_{1}, \ldots, q_{n}$ be BGPQs with the same arity and $\mathcal{R}$ a set of $R D F$ entailment rules.

- A generalization of $q_{1}, \ldots, q_{n}$ is a $B G P Q q_{g}$ such that $q_{i} \models_{\mathcal{R}} q_{g}$ for $1 \leq i \leq n$.

- A least general generalization of $q_{1}, \ldots, q_{n}$ is a generalization $q_{\operatorname{lgg}}$ of $q_{1}, \ldots, q_{n}$ such that for any other generalization $q_{g}$ of $q_{1}, \ldots, q_{n}: q_{1 \mathrm{gg}} \models_{\mathcal{R}} q_{g}$.

Unfortunately, this straightforward definition is of limited practical interest as the next example shows. Consider the BGPQs $q_{1}$ and $q_{2}$ in Figure 3, which respectively ask for the conference papers having some contact author, and for the journal papers having some author. Clearly, with the RDF entailment rules shown in Table 2 , an $\operatorname{lgg}$ of $q_{1}$ and $q_{2}$ is the very general BGPQ $q_{\operatorname{lgg}}(x) \leftarrow(x, \tau, y)$ asking for the resources having some type.

We argue that the value of lggs could be significantly augmented by taking into account some background knowledge formalized as ontological constraints. For example, if we consider the RDFS statements shown in Figure 3 that hold in the scientific publication domain, a more precise lgg for the above-mentioned $q_{1}, q_{2}$ would be $q_{\operatorname{lgg}}(x) \leftarrow(x, \tau$, Publication $),(x$, hasAuthor, $y),(y, \tau$, Researcher $)$ asking for the publications having some researcher as author, since $(i)$ having a contact author is having an author, (ii) only publications have authors, (iii) only researchers are authors, and (iv) conference and journal papers are publications.

To define such more precise lggs and state our learning problem in Section 3.2, we start by generalizing the standard specialization/generalization relation of entailment between BGPQs in Section 3.1, in order to allow comparing BGPQs w.r.t. an extra set of RDFS ontological constraints. In particular, this novel relation $(i)$ coincides with the standard one when extra constraints are unavailable and (ii) behaves like the standard one w.r.t. the central reasoning tasks of query entailment and of query answering when extra constraints are available. 


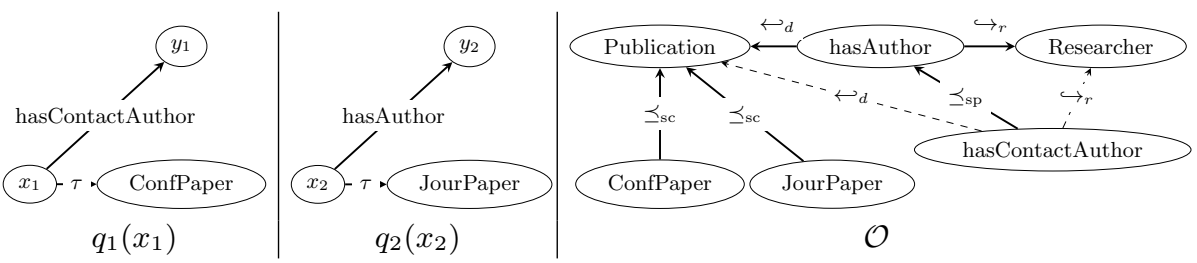

Fig. 3: Sample BGPQs $q_{1}$ and $q_{2}$; sample set $\mathcal{O}$ of RDFS ontological constraints.

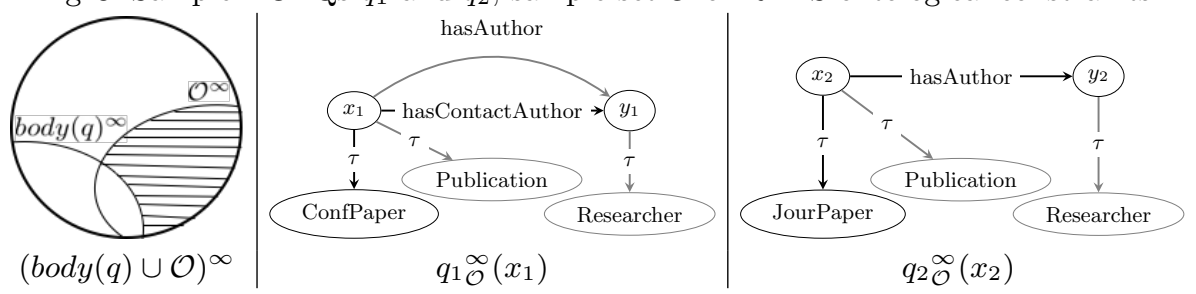

Fig. 4: Characterization of the body of a saturated BGPQ $q$ w.r.t. a set $\mathcal{O}$ of RDFS constraints (left), and saturations of $q_{1}$ and of $q_{2}$ w.r.t. $\mathcal{O}$ from Figure 3 (center and right respectively); triples shown in gray are added by saturation.

\subsection{Comparing Queries w.r.t. Ontological Constraints}

Our new entailment relation between queries builds on the following notion, which leverages the relevant background knowledge to complement a query:

Definition 2 (BGPQ saturation w.r.t. RDFS constraints). Let $\mathcal{R}$ be a set of RDF entailment rules, $\mathcal{O}$ a set of RDFS statements, and $q$ a BGPQ. The saturation of $q$ w.r.t. $\mathcal{O}$, noted $q_{\mathcal{O}}^{\infty}$, is the $B G P Q$ with the same answer variables as $q$ and whose body, noted body $\left(q_{\mathcal{O}}^{\infty}\right)$, is the maximal subset of $(\operatorname{bod} y(q) \cup \mathcal{O})^{\infty}$ such that for any of its subset $\mathcal{S}$ : if $\mathcal{O} \models_{\mathcal{R}} \mathcal{S}$ holds then body $(q) \models_{\mathcal{R}} \mathcal{S}$ holds.

In essence, the saturation of a BGPQ comprises all the triples in the saturation of its body together with the RDFS constraints, from which are pruned out the triples derived solely from the constraints, i.e., which are not related to what the query is asking for. This corresponds exactly to the non-hatched subset of $(\operatorname{bod} y(q) \cup \mathcal{O})^{\infty}$ shown in Figure 4: $\operatorname{bod} y\left(q_{\mathcal{O}}^{\infty}\right)=(\operatorname{bod} y(q) \cup \mathcal{O})^{\infty} \backslash\left(\mathcal{O}^{\infty} \backslash \operatorname{bod} y(q)^{\infty}\right)$. Of course, such a saturation is pertinent just in case the RDF entailment rules under consideration utilize the RDFS constraints, e.g., those in Table 2; otherwise the set of constraints is useless.

Figure 4 illustrates the saturation of queries w.r.t. ontological constraints using the BGPQs and RDFS contraints from Figure 3.

The next theorem states that a BGPQ and its saturation w.r.t. RDFS constraints are equivalent from the query entailment and query answering viewpoints:

Theorem 1. Let $\mathcal{R}$ be a set of RDF entailment rules, $\mathcal{O}$ a set of RDFS statements, and $q$ a BGPQ whose saturation w.r.t. $\mathcal{O}$ is $q_{\mathcal{O}}^{\infty}$. For any $R D F$ graph $\mathcal{G}$ whose set of RDFS statements is $\mathcal{O},(i) \mathcal{G} \models_{\mathcal{R}} q$ holds iff $\mathcal{G} \models_{\mathcal{R}} q_{\mathcal{O}}^{\infty}$ holds, and (ii) $q(\mathcal{G})=q_{\mathcal{O}}^{\infty}(\mathcal{G})$ holds.

We can now endow entailment between queries with background knowledge: 
Definition 3 (Entailment between BGPQs w.r.t. RDFS constraints). Given a set $\mathcal{R}$ of RDF entailment rules, a set $\mathcal{O}$ of RDFS statements, and two $B G P Q s q$ and $q^{\prime}$ with the same arity, $q$ entails $q^{\prime}$ w.r.t. $\mathcal{O}$, denoted $q \models_{\mathcal{R}, \mathcal{O}} q^{\prime}$, iff $q_{\mathcal{O}}^{\infty}=q^{\prime}$ holds.

Using the set $\mathcal{R}$ of entailment rules in Table 2 , the above mentioned BGPQ $q_{\operatorname{lgg}}(x) \leftarrow(x, \tau$, Publication), $(x$, hasAuthor, $y),(y, \tau$, Researcher $)$ is neither entailed by $q_{1}$ nor by $q_{2}$ from Figure 3 , while it is entailed by both of them w.r.t. the set $\mathcal{O}$ of constraints displayed in the same Figure, i.e., it is entailed in the standard fashion by their saturations shown in Figure 4: $q_{1} \mathcal{O}^{\infty} \models^{\phi_{1}} q$ holds for $\phi_{1}(x)=x_{1}$ and $\phi_{1}(y)=y_{1}$, and $q_{2} \underset{\mathcal{O}}{\infty} \models^{\phi_{2}} q$ holds for $\phi_{2}(x)=x_{2}$ and $\phi_{2}(y)=y_{2}$.

Clearly, the above definition coincides with standard RDF entailment between BGPQs when $\mathcal{O}$ is empty (recall Section 2). Further, the main theorem below states the required behaviour for a query entailed by another w.r.t. ontological constraints, i.e., the counterpart of Property 3 in Section 2: the former generalizes the latter from the query entailment and query answering viewpoints:

Theorem 2. Let $\mathcal{R}$ be a set of $R D F$ entailment rules, $\mathcal{O}$ a set of RDFS statements, and two BGPQs $q$ and $q^{\prime}$ such that $q \models_{\mathcal{R}, \mathcal{O}} q^{\prime}$. For any $R D F$ graph $\mathcal{G}$ whose set of RDFS statements is $\mathcal{O},(i)$ if $\mathcal{G} \models_{\mathcal{R}} q$ holds then $\mathcal{G} \models_{\mathcal{R}} q^{\prime}$ holds, and (ii) $q(\mathcal{G}) \subseteq q^{\prime}(\mathcal{G})$ holds.

\subsection{Learning lggs w.r.t. Ontological Contraints}

In the light of the preceding results, we revise/generalize Definition 1 as follows:

Definition 4 (lgg of BGPQs w.r.t. RDFS constraints). Let $\mathcal{R}$ be a set of $R D F$ entailment rules, $\mathcal{O}$ a set of RDFS statements, and $q_{1}, \ldots, q_{n} B G P Q s$ with the same arity.

- A generalization of $q_{1}, \ldots, q_{n}$ w.r.t. $\mathcal{O}$ is a $B G P Q q_{g}$ such that $q_{i} \models_{\mathcal{R}, \mathcal{O}} q_{g}$ for $1 \leq i \leq n$.

- $A$ least general generalization of $q_{1}, \ldots, q_{n}$ w.r.t. $\mathcal{O}$ is a generalization $q_{\mathrm{lgg}}$ of $q_{1}, \ldots, q_{n}$ w.r.t. $\mathcal{O}$ such that for any other generalization $q_{g}$ of $q_{1}, \ldots, q_{n}$ w.r.t. $\mathcal{O}: q_{\mathrm{lgg}}==_{\mathcal{R}, \mathcal{O}} q_{g}$.

By constrast with an lgg of RDF graphs that always exists [9], we found:

Theorem 3. An $\operatorname{lgg}$ of BGPQs w.r.t. RDFS statements may not exist for some set of RDF entailment rules; when it exists, it is unique up to entailment $(=\mathcal{R}, \mathcal{O})$.

Indeed, consider the BGPQs $q_{1}\left(x_{1}\right) \leftarrow\left(x_{1}\right.$, hasAuthor, $\left.y_{1}\right)$ asking for the resources having some author, and $q_{2}\left(x_{2}\right) \leftarrow\left(y_{2}\right.$, hasAuthor, $\left.x_{2}\right)$ asking for the authors of some resource. Clearly, when the set $\mathcal{R}$ of entailment rules is empty or comprises the rules in Table 2, no BGPQ can generalize $q_{1}$ and $q_{2}$, hence there is no lgg of them. By contrast, if we use the complete set of RDF entailment rules, an lgg of $q_{1}$ and $q_{2}$ is $q_{\operatorname{lgg}}(x) \leftarrow(x, \tau$, rdf:Resource), since every RDF value is an instance of the built-in class rdf:Resource. Also, when an lgg of BGPQs w.r.t. RDFS constraints exists, it is unique up to entailment, i.e., is 
semantically unique, because $q_{\operatorname{lgg}} \models_{\mathcal{R}, \mathcal{O}} q_{g}$ holds for any $q_{g}$ in Definition 1 . If it were that queries have multiples $\operatorname{lgg} s$ incomparable w.r.t. entailment, say the BGPQs $\operatorname{lgg}_{1}(\bar{x}), \ldots, \operatorname{lgg}_{m}(\bar{x})$, the BGPQ defined as $q_{\operatorname{lgg}}(\bar{x}) \leftarrow \operatorname{bod} y\left(\operatorname{lgg}_{1}\right) \cup \cdots \cup$ body $\left(\operatorname{lgg}_{m}\right)$ would be a single strictly more specific lgg, a contradiction.

Though unique up to entailment, there exist many syntactic variants (an infinity actually) of an lgg due to redundant triples, i.e., triples entailed by others within the lgg. For example, think of an $\operatorname{lgg} q_{\operatorname{lgg}}(x) \leftarrow(x, \tau, A),(x, \tau, B),(x, y, z)$ w.r.t. the set of constraints $\mathcal{O}=\left\{\left(A, \preceq_{\mathrm{sc}}, B\right),\left(B, \preceq_{\mathrm{sc}}, A\right)\right\}$, which asks for resources of types $A$ and $B$ that are somehow related to some resource, and it is known that $A$ and $B$ are equivalent classes. Clearly, different equivalent and minimal variants (w.r.t. the number of triples) of this $\operatorname{lgg}$ are $q_{\operatorname{lgg}}(x) \leftarrow(x, \tau, A)$ and $q_{\operatorname{lgg}}(x) \leftarrow(x, \tau, B)$, since $(x, y, z)$ is entailed by each of the two other triples, and $(x, \tau, B)$ is entailed by $(x, \tau, A)$ w.r.t. $\mathcal{O}$, and vice versa, because $A$ and $B$ are equivalent. Importantly, redundancy of triples is not specific to lggs of BGPQs w.r.t. RDFS constraints, since obviously any BGPQ may feature redundancy. The detection and elimination of such redundancy have been studied in the literature $[18,20]$, hence we focus in this work on learning some lgg of BGPQs w.r.t. RDFS constraints; learning as minimal as possible lggs is a perspective of this work discussed in Section 6.

Based on the above discussion, the learning problem we propose to study is:

Problem 1. Given a set $\mathcal{R}$ of RDF entailment rules, a set $\mathcal{O}$ of RDFS statements, and the BGPQs $q_{1}, \ldots, q_{n}$ with the same arity, find an $\operatorname{lgg}$ of $q_{1}, \ldots, q_{n}$ w.r.t. $\mathcal{O}$.

Importantly, the proposition below shows that an lgg of $n \geq 3$ BGPQs can be inductively defined, hence computed, as a sequence of $n-1$ lggs of two BGPQs. That is, assuming that $\ell_{k \geq 2}$ is an operator computing an lgg of $k$ input BGPQs, the next proposition establishes that:

$$
\begin{aligned}
& \text { [basis] } \ell_{3}\left(q_{1}, q_{2}, q_{3}\right) \equiv_{\mathcal{R}, \mathcal{O}} \ell_{2}\left(\ell_{2}\left(q_{1}, q_{2}\right), q_{3}\right) \\
& \text { [induction] } \ell_{n}\left(q_{1}, \ldots, q_{n}\right) \equiv_{\mathcal{R}, \mathcal{O}} \ell_{2}\left(\ell_{n-1}\left(q_{1}, \ldots, q_{n-1}\right), q_{n}\right) \\
& \equiv_{\mathcal{R}, \mathcal{O}} \ell_{2}\left(\ell_{2}\left(\cdots \ell_{2}\left(\ell_{2}\left(q_{1}, q_{2}\right), q_{3}\right) \cdots, q_{n-1}\right), q_{n}\right)
\end{aligned}
$$

Proposition 1. Let $q_{1}, \ldots, q_{n \geq 3}$ be $n$ BGPQs, $\mathcal{O}$ a set of RDFS statements and $\mathcal{R}$ a set of RDF entailment rules. $q_{\operatorname{lgg}}$ is an $\operatorname{lgg}$ of $q_{1}, \ldots, q_{n}$ w.r.t. $\mathcal{O}$ iff $q_{\operatorname{lgg}}$ is an $\operatorname{lgg}$ w.r.t. $\mathcal{O}$ of an $\operatorname{lgg}$ of $q_{1}, \ldots, q_{n-1}$ w.r.t. $\mathcal{O}$ and $q_{n}$.

Based on the above result, without loss of generality, we study in the next Section the particular instance of our learning problem for $n=2$.

\section{Computing lggs of queries w.r.t. ontological constraints}

Our solution to the above learning problem (Problem 1) builds on the notion of least general anti-unifier of two atoms [21,23], which is dual to the well-known notion of most general unifier of two atoms [22,23]. We use it to devise the cover query of two BGPQs $q_{1}$ and $q_{2}$ (to be defined shortly, Definition 5 below), which is an lgg of $q_{1}$ and $q_{2}$ just in case both RDF entailment rules and ontological constraints are ignored (Theorem 4). Further, we show (Theorem 5) that an 
lgg of $q_{1}$ and $q_{2}$ as defined in Definition 4, i.e., when RDF entailment rules and ontological constraints are taken into consideration, is the cover query of the saturations of $q_{1}$ and of $q_{2}$ with the RDF entailment rules and ontological constraints at hand (Definition 2). We also provide the size of these cover querybased lggs (i.e., number of triples), as well as the time to compute them.

Definition 5 (Cover query). Let $q_{1}, q_{2}$ be two BGPQs with the same arity $n$. If there exists the $B G P Q q$ such that

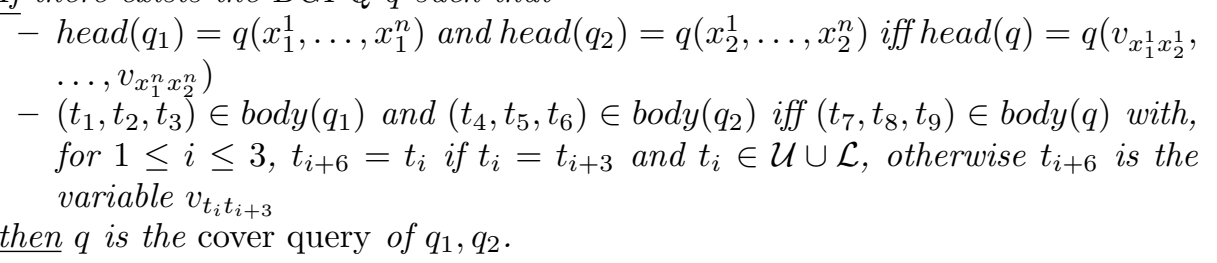

The rationale behind the above definition of cover query is that $(i) q$ 's head is defined as the least general anti-unifier of the heads of $q_{1}$ and $q_{2}$ (first item above) and ( $i$ i) each $q$ triple is defined as a least general anti-unifier of an explicit $q_{1}$ triple and an explicit $q_{2}$ triple (second item above), so that, when the cover query exits (If ...then ... above), it is a generalization of $q_{1}$ and $q_{2}$ just in case RDF entailment rules and ontological constraints are not considered (first item in Definition 4 with $\mathcal{R}=\emptyset$ and $\mathcal{O}=\emptyset$ ). Moreover, crucially, $(\mathrm{iii})$ the variables used to generalize pairs of distinct values across all the anti-unifications begetting $q$ are consistently named: each time the distinct values $\alpha$ from $q_{1}$ and $\beta$ from $q_{2}$ are generalized by a variable across these anti-unifications, it is always by the same $q$ variable $v_{\alpha \beta}$. This naming scheme enforces joins between $q$ triples, which capture the common join structure within $q_{1}$ and $q_{2}$, so that $q$ is not only a generalization of $q_{1}$ and $q_{2}$ but also a least general generalization of them (second item in Definition 4 with $\mathcal{R}=\emptyset$ and $\mathcal{O}=\emptyset$ ).

The cover query $q$ of the BGPQs $q_{1}$ and $q_{2}$ is displayed in Figure 5 (top). Its triple $\left(v_{x_{1} x_{2}}, \tau, v_{\mathrm{CPJP}}\right)$ results from anti-unifying the $q_{1}$ triple $\left(x_{1}, \tau\right.$, ConfPaper $)$ and the $q_{2}$ triple $\left(x_{2}, \tau\right.$, JourPaper); the variable $v_{x_{1} x_{2}}$ is the least general value for the subject values $x_{1}$ and $x_{2}$, the URI $\tau$ is that for the property values $\tau$ (because a constant is the least generalization of itself), and the variable $v_{\text {CPJP }}$ is that for the object values ConfPaper and JourPaper. This $q$ triple captures that $q_{1}$ and $q_{2}$ both ask for resources having some type. Here, the fact this type is related to scientific publications is missed, due to the absence of background knowledge relating conference papers, journal papers and scientific publications. Similarly, the $q$ triple $\left(v_{x_{1} x_{2}}, v_{\mathrm{hCAhCA}}, v_{y_{1} y_{2}}\right)$ results from anti-unifying the $q_{1}$ triple $\left(x_{1}\right.$, hasContactAuthor, $\left.y_{1}\right)$ and the $q_{2}$ triple $\left(x_{2}\right.$, hasAuthor, $\left.y_{2}\right)$. Because of our consistent naming of variables within $q$, this $q$ triple and the preceding one join on $v_{x_{1} x_{2}}$. Unfortunately, this second triple does not enhance the description of $v_{x_{1} x_{2}}$ in $q$, since it generalizes, hence is redundant with, the preceding one. It only captures from $q_{1}$ and $q_{2}$ that $q$ asks for resources having somehow related to something. Here again, the fact that this relationship is to have some author is missed due to the absence of background knowledge. The two other antiunifications begetting $q$ 's body also produce redundant triples. 


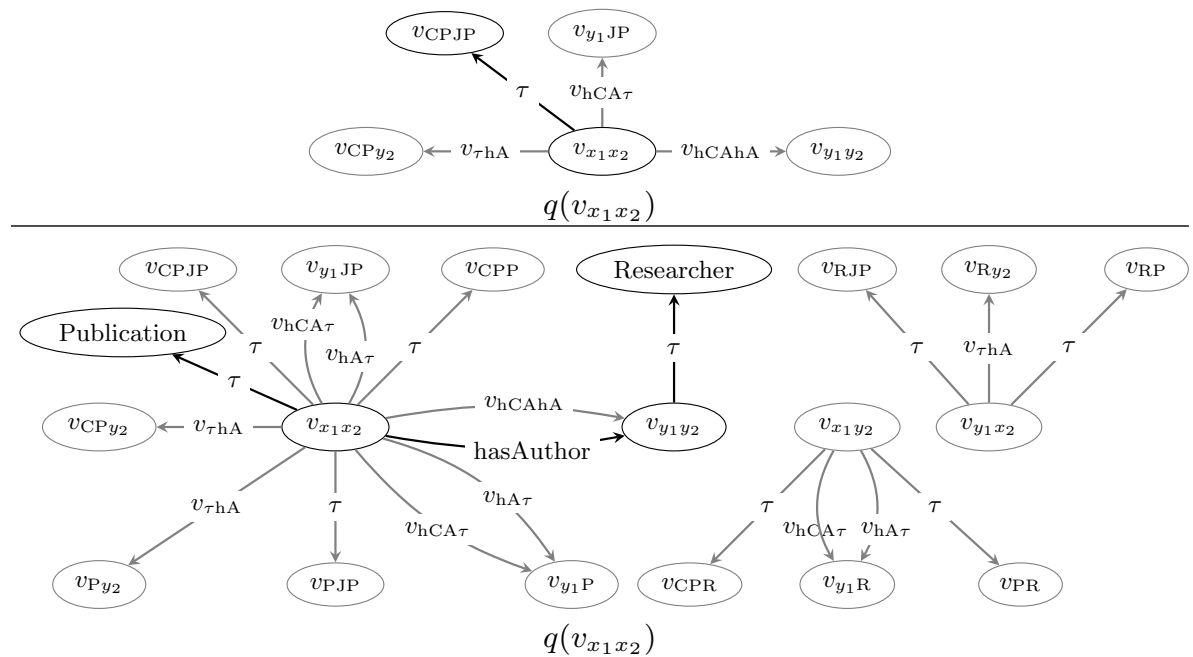

Fig. 5: Cover queries of the BGPQs $q_{1}$ and $q_{2}$ in Figure 3 (top) and of their saturations $q_{1} \stackrel{\infty}{\mathcal{O}}$ and $q_{2} \underset{\mathcal{O}}{\infty}$ in Figure 4 (bottom). Triples in grey are redundant w.r.t. those in black.

As mentioned earlier, the cover query $q$ of two BGPQs $q_{1}$ and $q_{2}$ may not exist. This happens when $q$, as defined in Definition 5, has its head not compatible with its body: some required answer variable(s) cannot be supplied by $q$ 's body. For instance, recall the BGPQs $q_{1}$ and $q_{2}$ used in Section 3.2 to point out that an lgg may no exist. Their cover query does not exist either, because Definition 5 leads to $q\left(v_{x_{1} x_{2}}\right) \leftarrow\left(v_{x_{1} y_{2}}\right.$, hasAuthor, $\left.v_{y_{1} x_{2}}\right)$, which is not a BGPQ (the answer variable $v_{x_{1} x_{2}}$ does not appear in the body). Importantly, the existence of an lgg of BGPQs and the existence of their cover query coincide.

The next theorem formalizes the above discussion:

Theorem 4. Given two BGPQs $q_{1}, q_{2}$ with the same arity and empty sets $\mathcal{R}$ of $R D F$ entailment rules and $\mathcal{O}$ of RDFS statements:

1. the cover query of $q_{1}$ and $q_{2}$ exists iff an $\operatorname{lgg}$ of $q_{1}$ and $q_{2}$ exists;

2. the cover query of $q_{1}$ and $q_{2}$ is an $\operatorname{lgg}$ of $q_{1}$ and $q_{2}$.

It follows from the above result that the cover query $q$ of two BGPQs $q_{1}$ and $q_{2}$ displayed in Figure 5 (top) is an lgg of them just in case both RDF entailment rules and extra RDFS ontological constraints are ignored.

We provide below the worst-case time to compute a cover query, and its size.

Proposition 2. The cover query of two BGPQs $q_{1}$ and $q_{2}$ can be computed in $O\left(\left|\operatorname{body}\left(q_{1}\right)\right| \times\left|\operatorname{bod} y\left(q_{2}\right)\right|\right)$; its size is $\left|\operatorname{body}\left(q_{1}\right)\right| \times\left|\operatorname{bod} y\left(q_{2}\right)\right|$.

The next theorem generalizes the preceding one in order to use the notion of cover query to compute an lgg of two queries w.r.t. extra RDFS ontological constraints and any set of RDF entailment rules.

Theorem 5. Given a set $\mathcal{R}$ of RDF entailment rules, a set $\mathcal{O}$ of RDFS statements and two BGPQs $q_{1}, q_{2}$ with the same arity,

1. the cover query $q$ of $q_{1}{ }_{\mathcal{O}}^{\infty}, q_{2}{ }_{\mathcal{O}}^{\infty}$ exists iff an $\operatorname{lgg}$ of $q_{1}, q_{2}$ w.r.t. $\mathcal{O}$ exists;

2. the cover query $q$ of $q_{1} \mathcal{O}^{\infty}, q_{2} \mathcal{O}$ is an $\operatorname{lgg}$ of $q_{1}, q_{2}$ w.r.t. $\mathcal{O}$. 
As an immediate consequence of the above results, we get the following worstcase time to compute an lgg of two BGPQs $q_{1}$ and $q_{2}$, and its size. We assume given the saturation $q_{1} \mathcal{O}^{\infty}$ and $q_{2} \mathcal{O}_{\mathcal{O}}^{\infty}$ w.r.t. the sets $\mathcal{O}$ of RDFS constraints and $\mathcal{R}$ of RDF entailment rules under consideration, as the times to compute $q_{1}^{\infty} \infty$ and $q_{2} \mathcal{O}$, and their sizes, depend on the particular sets $\mathcal{O}$ and $\mathcal{R}$ at hand.

Corollary 1. A cover query-based lgg of two BGPQs $q_{1}$ and $q_{2}$ is computed in $O\left(\left|\operatorname{body}\left(q_{1 \mathcal{O}}^{\infty}\right)\right| \times\left|\operatorname{bod} y\left(q_{2} \underset{\mathcal{O}}{\infty}\right)\right|\right)$ and its size is $\left|\operatorname{body}\left(q_{1_{\mathcal{O}}}^{\infty}\right)\right| \times\left|\operatorname{body}\left(q_{2}{ }_{\mathcal{O}}^{\infty}\right)\right|$.

Figure 5 (bottom) displays the cover query of the BGPQs $q_{1} \mathcal{O}_{\mathcal{O}}^{\infty}$ and $q_{2} \mathcal{O}$ shown in Figure 4. It is therefore (Theorem 5) an lgg of the BGPQs $q_{1}$ and $q_{2}$ w.r.t. the set $\mathcal{O}$ of RDFS constraints, all shown in Figure 3, using the RDF entailment rules shown in Table 2.

Figure 5 exemplifies the benefits of taking into account extra ontological constraints modeling background knowledge when identifying the commonalities between queries, thus of endowing the RDF relation of generalization/specialization between queries with such knowledge. When background knowledge is ignored (top), we only learn that both $q_{1}$ and $q_{2}$ ask for the resources having some type. In contrast, when we do consider background knowledge (bottom), we further learn that these resources, which both $q_{1}$ and $q_{2}$ ask for, are publications, which have some researcher as author.

\section{Experiments}

Goal. We study the added-value of considering background knowledge when learning lggs of queries. As Proposition 3 shows below, this amounts to measuring how much more precise is an lgg of queries that considers background knowledge than an lgg of the same queries that ignores background knowledge:

Proposition 3. Given a set $\mathcal{R}$ of RDF entailment rules, a set $\mathcal{O}$ of RDFS statements, two BGPQs $q_{1}, q_{2}$ with the same arity, an $\operatorname{lgg} q_{\operatorname{lgg}}$ of $q_{1}, q_{2}$ (Definition 1) and an $\operatorname{lgg} q_{\operatorname{lgg}}^{\mathcal{O}}$ of $q_{1}, q_{2}$ w.r.t. $\mathcal{O}$ (Definition 4), $q_{\operatorname{lgg}}^{\mathcal{O}} \models_{\mathcal{R}} q_{\operatorname{lgg}}$ holds.

Intuitively, this result follows from the fact that $(i) q_{\operatorname{lgg}}$ is equivalent to the cover query-based lgg $q$ of the saturations of $q_{1}$ and of $q_{2}$ w.r.t. the empty set of RDFS constraints, (ii) $q_{\mathrm{lgg}}^{\mathcal{O}}$ is equivalent to the cover query-based lgg $q^{\prime}$ of the saturations of $q_{1}$ and of $q_{2}$ w.r.t. $\mathcal{O}$, and (iii) by definition of a cover query (Definition 5), $q$ and $q^{\prime}$ have the same heads and the body of $q$ is a subset of that $q^{\prime}$, thus $q^{\prime} \models_{\mathcal{R}} q$ holds, hence $q_{1 \mathrm{gg}}^{\mathcal{O}} \models_{\mathcal{R}} q_{\mathrm{lgg}}$ holds.

From this result and Property 3 (Section 2.2), $q_{\mathrm{lgg}}^{\mathcal{O}}(\mathcal{G}) \subseteq q_{\mathrm{lgg}}(\mathcal{G})$ holds for any RDF graph $\mathcal{G}$, and clearly the more $q_{\mathrm{lgg}}^{\mathcal{O}}$ is specific w.r.t. $q_{\mathrm{lgg}}$, the smaller the subset $q_{\operatorname{lgg}}^{\mathcal{O}}(\mathcal{G})$ of $q_{\operatorname{lgg}}(\mathcal{G})$ is, i.e., the smaller $\left|q_{\operatorname{lgg}}^{\mathcal{O}}(\mathcal{G})\right|$ is w.r.t. $\left|q_{\operatorname{lgg}}(\mathcal{G})\right|$. Therefore, as a practical metric for measuring the semantic distance between $q_{\operatorname{lgg}}^{\mathcal{O}}$ and $q_{\operatorname{lgg}}$ through $\models_{\mathcal{R}}$, we compute the gain in precision in (\%) that background knowledge yields w.r.t. query answering as:

gain in precision $=1-\frac{\left|q_{\operatorname{lgg}}^{\mathcal{O}}(\mathcal{G}) \cap q_{\lg }(\mathcal{G})\right|}{\left|q_{\operatorname{lgg}}(\mathcal{G})\right|}=1-\frac{\left|q_{\operatorname{lgg}}^{\mathcal{O}}(\mathcal{G})\right|}{\left|q_{\operatorname{lgg}}(\mathcal{G})\right|}$ since $q_{\operatorname{lgg}}^{\mathcal{O}}(\mathcal{G}) \subseteq q_{\operatorname{lgg}}(\mathcal{G})$. 
Prototype. We implemented our technical contributions in Java 1.8, on top of the Jena 3.0.1 RDF reasoner and of a PostgreSQL 9.3.11 server, all used with default settings; our implemented algorithms are detailed in [8].

We used Jena to compute the saturation of an RDF graph, against which queries must be evaluated to obtained their complete answer sets (Section 2.1). We also used Jena to compute the saturation $q_{\mathcal{O}}^{\infty}$ of a $B G P Q$ q w.r.t. a set $\mathcal{O}$ of RDFS constraints (Definition 2): we rely on Jena's saturation, union and difference operators to compute $q_{\mathcal{O}}^{\infty}$ 's body as described in Section 3.1.

We used PostgreSQL to evaluate SQLized BGPQs against a saturated RDF graph stored in a Triple (s,p,o) table.

We deployed our prototype on an Intel Xeon X5550 2.67GHz machine with 32GB of RAM, running Ubuntu 14.04.3 64bits; times reported below are in ms.

Setting. We conducted experiments using real DBpedia data [17] and synthetic LUBM data [12]. For space reasons, we present only our DBpedia experiments; LUBM ones can be found in [8] and allow drawing similar conclusions.

We used the subset of standard RDF entailment rules in Table 2, which fully allows exploiting RDFS ontological constraints, i.e., background knowledge.

From the DBpedia dataset, we picked four complementary files ${ }^{1}$ to build the $\mathrm{RDF}$ graph $\mathcal{G}_{\text {DBpedia }}$ comprising $41.18 \mathrm{M}$ triples, whose subset $\mathcal{O}_{\text {DBpedia }}$ of $30.31 \mathrm{k}$ RDFS constraints represents DBpedia's background knowledge. The saturation of $\mathcal{G}_{\text {DBpedia }}$ comprises $78.14 \mathrm{M}$ triples and takes about 30 minutes to be computed.

Finally, we defined 42 test BGPQs, among which we picked 8 representative ones with 2 variables; they can be found in [8]. Table 3 displays their characteristics (top), as well as their saturation size and time (bottom): the size augments from $\times 3.16$ for $Q_{2}$ up to $\times 4.75$ for $Q_{3}$; the time is $692 \mathrm{~ms}$ on average. Also, importantly, queries $Q_{1}-Q_{4}$ (left) are heterogeneous in the sense that they differ significantly both on their structure and the kind of information they ask for, hence use many distinct classes, properties and URI values, while $Q_{4}-Q_{8}$ (right) are homogeneous and only differ in some classes, properties and URI values.

Results. First, as Table 4 (lines 1 and 3) shows, the cover query-based lggs of test queries are always computed fast whether or not the DBpedia constraints are considered: from 3 to $6 \mathrm{~ms}$ when ignored, to 13 to $18 \mathrm{~ms}$ when considered.

Table 4 (lines 2 and 4 ) also shows that the answer set of an lgg is significantly larger when DBpedia constraints are not taken into account: the size difference goes from a small $\times 1.02$ for the homogeneous queries $Q_{5}, Q_{7}$ up to a striking $\times 76.42$ for the heterogeneous queries $Q_{1}, Q_{4}$, with a significant average of $\times 17.38$ $(\times 33.34$ for the heterogeneous queries and $\times 1.42$ for the homogeneous ones). This translates into the precision gains shown at line $5: 58 \%$ overall, $90 \%$ for the heterogeneous queries, and $25 \%$ for the homogeneous ones.

These results confirm our claim that taking into account background knowledge yields more precise lggs. Indeed, ontological constraints help finding common super- classes and properties to be used in lggs in place of the different ones used in input queries; when constraints are ignored, these can just be generalized

\footnotetext{
${ }^{1}$ We use the dbpedia_2015-10.nt RDF Schema file and the instance_types_en.ttl, mappingbased_literals_en.ttl and mappingbased_objects_en.ttl RDF data files.
} 


\begin{tabular}{|l||c|c|c||c||c|c|c|c|}
\hline Query $Q_{1 \leq i \leq 8}:$ & $Q_{1}$ & $Q_{2}$ & $Q_{3}$ & $Q_{4}$ & $Q_{5}$ & $Q_{6}$ & $Q_{7}$ & $Q_{8}$ \\
\hline \hline \hline$Q_{i}{ }^{\prime}$ s shape & tree & tree & tree & graph & graph & graph & graph & graph \\
\hline $\mid$ body $\left(Q_{i}\right) \mid$ & 4 & 6 & 4 & 6 & 4 & 6 & 6 & 6 \\
\hline Number of URI/variable occurrence in $Q_{i}$ & $7 / 5$ & $9 / 9$ & $5 / 7$ & $7 / 11$ & $5 / 7$ & $9 / 9$ & $9 / 9$ & $9 / 9$ \\
\hline$\left|Q_{i}\left(\mathcal{G}_{\text {DBpedia }}\right)\right|$ & 77 & 0 & 41695 & 13 & 6 & 0 & 1 & 0 \\
\hline \hline $\mid$ body $\left(Q_{i}{ }_{\mathcal{O}_{\text {DBpedia }}}\right) \mid$ & 16 & 19 & 19 & 23 & 16 & 23 & 23 & 23 \\
\hline Time to compute $Q_{i}{ }_{\mathcal{O}_{\text {DBpedia }}^{\infty}}^{\infty}$ & 666 & 643 & 677 & 734 & 681 & 706 & 697 & 736 \\
\hline
\end{tabular}

Table 3: Characteristics of our test BGPQs (top) and of their saturations (bottom).

\begin{tabular}{|c|c|c|c|c|c|c|c|c|}
\hline lgg of: & $Q_{1} Q_{2}$ & $Q_{1} Q_{3}$ & $Q_{1} Q_{4}$ & $Q_{2} Q_{3}$ & \multicolumn{4}{|c|}{\begin{tabular}{|l|l|l|l}
$Q_{4} Q_{5}$ & $Q_{5} Q_{6}$ & $Q_{5} Q_{7}$ & $Q_{7} Q_{8}$ \\
\end{tabular}} \\
\hline Time to comp & 3 & 3 & 5 & 4 & $\overline{4}$ & 5 & $\overline{6}$ & 5 \\
\hline$\left|q_{\text {lgg }}\left(\mathcal{G}_{\text {DBpedia }}\right)\right|$ & 477,455 & $34,747,102$ & $34,901,117$ & $60,356,807$ & 1,977 & 1221 & 35 & 70 \\
\hline Time to compute $q_{1 \mathrm{gg}}^{\mathcal{O}_{\text {Dpedia }}}$ & 13 & 14 & 14 & 15 & 15 & 14 & 17 & 18 \\
\hline$\left|q_{1 \mathrm{gBg}}^{\mathcal{O}_{\text {Dedia }}}\left(\mathcal{G}_{\text {DBpedia }}\right)\right|$ & 10,637 & $7,874,768$ & 456,690 & $7,874,768$ & $\mid 1,701$ & 780 & 34 & 36 \\
\hline Gain in precision & 98 & 77 & 99 & 87 & 14 & 36 & 3 & 49 \\
\hline
\end{tabular}

Table 4: Characteristics of cover query-based lggs of test queries, w/ or w/o using the DBpedia RDFS constraints.

using variables. Therefore, the more heterogeneous input queries are, the more such common super-classes and properties are used in their lgg instead of variables, and the more the gain in precision of their lgg is high. For homogeneous input queries, while less striking, the gain in precision is significant in general.

\section{Related work and conclusion}

The reasoning problem of learning lggs has been studied in various formalisms, e.g., Conceptual Graphs (CGs), Description Logics (DLs), RDF and SPARQL.

Most of the solutions exploit the (underlying) structure of the input descriptions, like trees for DL formulae (e.g., $[1,14,27])$ and for unary tree-shaped BGPQs $[2,16]$, and directed single-root graphs for the RDF $r$-graphs of [7]. Roughly speaking, they all consist in a simultaneous traversal of the input descriptions, starting from their roots, while incrementally computing their lgg. In contrast, when the input descriptions do not have a particular (or imposed) structure, solutions need to blindly traverse them while still being able to compute their lgg. They rely on standard categorial graph product for the so-called simple (i.e., purely conjunctive) CGs [3], on anti-unifications of triples for general RDF graphs [9], and on anti-unifications of query heads and of query body triples for the general BGPQs considered in this paper. Further, while (some of) the above solutions take into account background knowledge in CGs, DLs, and RDF, this is not the case for the state of the art in SPARQL $[2,16]$ : unary tree-shaped BGPQs are solely compared based on standard graph homomorphism $\left(\models_{\emptyset}\right)$.

Our results significantly advance the state of the art $[2,16]$ by considering (i) general BGPQs and (ii) background knowledge to obtain more precise lggs, as our experiments showed. Next, we plan studying heuristics that prune out as much as possible redundant triples, while computing lggs. Indeed, as Figure 5 shows, our cover query-based lggs may contain redundant triples. This would allow having more compact lggs, as well as reducing the a posteriori elimination effort of redundant triples using standard technique from the literature. 


\section{References}

1. Baader, F., Sertkaya, B., Turhan, A.Y.: Computing the least common subsumer w.r.t. a background terminology. Journal of Applied Logic 5(3), 392 - 420 (2007)

2. Bühmann, L., Lehmann, J., Westphal, P.: DL-Learner - a framework for inductive learning on the Semantic Web. Journal of Web Semantics 39, 15-24 (2016)

3. Chein, M., Mugnier, M.: Graph-based Knowledge Representation - Computational Foundations of Conceptual Graphs. Springer (2009)

4. Chuang, S.L., Chien, L.F.: Towards automatic generation of query taxonomy: A hierarchical query clustering approach. In: ICDM (2002)

5. Cohen, W.W., Borgida, A., Hirsh, H.: Computing least common subsumers in description logics. In: AAAI (1992)

6. Colazzo, D., Goasdoué, F., Manolescu, I., Roatis, A.: RDF analytics: lenses over semantic graphs. In: WWW (2014)

7. Colucci, S., Donini, F.M., Giannini, S., Sciascio, E.D.: Defining and computing least common subsumers in RDF. Journal of Web Semantics 39, 62-80 (2016)

8. El Hassad, S., Goasdoué, F., Jaudoin, H.: Learning commonalities in RDF and SPARQL (research report). https://hal.inria.fr/hal-01386237 (2016)

9. El Hassad, S., Goasdoué, F., Jaudoin, H.: Learning commonalities in RDF. In: ESWC (2017)

10. El Hassad, S., Goasdoué, F., Jaudoin, H.: Towards learning commonalities in SPARQL. In: ESWC (2017), poster track

11. Goasdoué, F., Karanasos, K., Leblay, J., Manolescu, I.: View selection in semantic web databases. PVLDB 5(2), 97-108 (2011)

12. Guo, Y., Pan, Z., Heflin, J.: LUBM: A benchmark for OWL knowledge base systems. Journal of Web Semantics 3(2-3), 158-182 (Oct 2005)

13. Huang, Z., Cautis, B., Cheng, R., Zheng, Y.: Kb-enabled query recommendation for long-tail queries. In: CIKM (2016)

14. Küsters, R.: Non-Standard Inferences in Description Logics, LNCS, vol. 2100. Springer (2001)

15. Le, W., Kementsietsidis, A., Duan, S., Li, F.: Scalable multi-query optimization for SPARQL. In: ICDE (2012)

16. Lehmann, J., Bühmann, L.: Autosparql: Let users query your knowledge base. In: ESWC (2011)

17. Lehmann, J., Isele, R., Jakob, M., Jentzsch, A., Kontokostas, D., Mendes, P.N., Hellmann, S., Morsey, M., van Kleef, P., Auer, S., Bizer, C.: DBpedia. Semantic Web 6(2), 167-195 (2015)

18. Meier, M.: Towards rule-based minimization of RDF graphs under constraints. In: RR (2008)

19. Picalausa, F., Luo, Y., Fletcher, G.H., Hidders, J., Vansummeren, S.: A structural approach to indexing triples. In: ESWC (2012)

20. Pichler, R., Polleres, A., Skritek, S., Woltran, S.: Complexity of redundancy detection on RDF graphs in the presence of rules, constraints, and queries. Semantic Web 4(4), 351-393 (2013)

21. Plotkin, G.D.: A note on inductive generalization. Machine Intelligence 5 (1970)

22. Robinson, J.A.: A machine-oriented logic based on the resolution principle. J. ACM 12(1), 23-41 (Jan 1965)

23. Robinson, J.A., Voronkov, A. (eds.): Handbook of Automated Reasoning. Elsevier and MIT Press (2001)

24. Resource Description Framework 1.1. https://www.w3.org/TR/rdf11-concepts

25. RDF 1.1 Semantics. https://www.w3.org/TR/rdf11-mt/

26. SPARQL 1.1. https://www.w3.org/TR/sparql11-query/

27. Zarrieß, B., Turhan, A.: Most specific generalizations w.r.t. general EL-TBoxes. In: IJCAI (2013) 